\title{
Discussão de alguns condicionantes da eficiência em universidades federais brasileiras a partir do Reuni
}

\author{
Ney Paulo Moreira \\ Universidade Federal de Viçosa (UFV)
}

Gideon Carvalho de Benedicto

Universidade Federal de Lavras (UFLA)

Francisval de Melo Carvalho

Universidade Federal de Lavras (UFLA)

Busca-se desenvolver uma análise do desempenho das universidades federais brasileiras que aderiram ao Reuni, bem como identificar os fatores condicionantes da eficiência, no período de 2008 a 2015. A análise por envoltória de dados (DEA) foi empregada na mensuração da eficiência relativa e foi utilizado o modelo de regressão Tobit, com dados em painel para a determinação dos fatores associados à eficiência. Verificou-se que, em média, as universidades federais brasileiras atuaram abaixo de seu potencial, apresentando uma queda no desempenho, especialmente no ano de 2009, com recuperação gradativa nos anos seguintes. Pôde-se notar que, sob diversos aspectos, como a relação alunos por professor e a proporção do atendimento a alunos de graduação e pós-graduação, as universidades tornaram-se um pouco mais homogêneas. As maiores relações de alunos por professor e alunos por servidor apresentaram relação positiva com o desempenho, assim como o tamanho das universidades mostrou-se positivamente associado com os mais altos escores de eficiência. No entanto, a quantidade de campi fora da sede apresentou relação negativa com a eficiência das universidades federais brasileiras.

Palavras-chave: eficiência, indicadores de desempenho, Reuni.

[Artigo recebido em 6 de junho de 2018. Aprovado em 12 de agosto de 2019.] 


\section{Discussion of some efficiency conditioning factors in Brazilian federal universities based on Reuni}

The study was performed aiming to carry out a performance analysis for Brazilian federal universities subscribed to the Reuni, as well as to identify efficiency conditioning factors for the period from 2008 to 2015. The data envelopment analysis was carried out to measure the relative efficiency, and the Tobit model was used to determine factors associated with the efficiency. Results show that Brazilian federal universities operated below their potential in the period under study, showing a decrease in performance over time, especially in 2009, with gradual recovery in the following years. By analyzing the student-teacher ratio and the service given by teachers to students at master's and doctoral degrees, universities appeared to become somewhat more uniform. The greater ratio student-teacher and student-servant showed a positive relationship with the performance, as well as the association between the size of universities with the higher efficiency score. The quantity of campuses found out of headquarters showed a negative relationship with the efficiency of universities under study.

Keywords: efficiency, performance indicators, Reuni.

\section{Discusión de algunos factores condicionantes de la eficiencia en las universidades federales brasileñas a partir del Reuni}

Búsqueda desarrollarse un análisis del desempeño de las universidades federales brasileñas que adhirieron al Reuni, así como identificar los factores condicionantes de la eficiencia, en el periodo de 2008 a 2015. El análisis por envolvente de datos (DEA) fue empleada en la medición de la eficiencia relativa y fue utilizado el modelo de regresión Tobit con datos en panel para la determinación de los factores asociados a la eficiencia. Se verificó que, en media, las universidades federales brasileñas actuaron abajo de su potencial, presentando una caída en el rendimiento, especialmente el año de 2009, con recuperación gradual en los años siguientes. Se pudo notar que, bajo diversos aspectos, como la relación alumnos por profesor y la proporción de la atención a alumnos de graduación y postgraduación, las universidades se hicieron un poco más homogéneas. Las mayores relaciones de alumnos por profesor y alumnos por servidor presentaron relación positiva con el desempeño, así como el tamaño de las universidades se mostró positivamente asociado con las más altas puntuaciones de eficiencia. Sin embargo, la cantidad de campi fuera de la sede presentó relación negativa con la eficiencia de las universidades federales brasileñas.

Palabras clave: eficiencia, indicadores de desempeño, Reuni. 


\section{Introdução}

As universidades federais brasileiras passam, atualmente, por um processo que tem gerado profundas transformações decorrentes de políticas que visam à expansão e democratização do acesso ao ensino superior. A ampliação desse nível de ensino é importante, tendo em vista que, assim como ocorre em outros países (PORTO; RÉGNIER, 2003), o sistema educacional do Brasil tem se mostrado incapaz de absorver grande parte dos concluintes do ensino secundário.

Nesse sentido, considerando a meta de expansão da oferta de educação superior constante no Plano Nacional de Educação, foi instituído pelo Decreto no 6.096/2007 (BRASIL, 2007) o Programa de Apoio a Planos de Reestruturação e Expansão das Universidades Federais (Reuni), com o objetivo de "criar condições para a ampliação do acesso e permanência na educação superior, no nível de graduação, pelo melhor aproveitamento da estrutura física e de recursos humanos existentes nas universidades federais".

O Reuni contou com a adesão de 53 universidades federais, sendo que os planos de 21 delas foram implantados no primeiro semestre de 2008, e os demais no segundo semestre do mesmo ano (BRASIL, 2009). O programa apresentou como meta global a elevação gradual da taxa de conclusão média dos cursos de graduação presenciais para noventa por cento, bem como o aumento da relação de alunos de graduação por professor para dezoito, ao final de cinco anos, a contar do início de cada plano.

Sendo de competência da União organizar, manter e desenvolver os órgãos e instituições do sistema federal de ensino (BRASIL, 1996), dentre elas, as universidades federais, tais políticas de expansão acabam gerando acréscimos na demanda por financiamento público, tornando ainda mais evidente a necessidade do emprego de mecanismos que visem garantir a utilização eficiente desses recursos. Em sua própria formulação, o Reuni demonstra a preocupação com a eficiência dos gastos públicos ao estabelecer como objetivo indireto, ou secundário, o melhor aproveitamento dos recursos das universidades federais, visando otimizar a estrutura pública.

Portanto, avaliar o desempenho das instituições públicas de ensino superior torna-se essencial ao passo que oferece informações que podem contribuir no processo de tomada de decisões, direcionando-as a uma gestão eficiente. Contudo, segundo Belloni, Magalhães e Sousa (2003), ainda se percebe um grande amadorismo nas práticas de avaliação institucional e de políticas públicas em educação. Feldmann e Souza (2016) relatam que as práticas atuais de avaliação são pautadas em uma lógica econômica que produz apenas efeitos de regulação e muitas vezes gera resultados conflituosos. 
Na literatura acadêmica, percebe-se que a alocação de recursos públicos na educação tem gerado muitos estudos no mundo todo, principalmente aqueles que visam mensurar a eficiência de instituições de ensino superior e ranqueá-las. Dentre os métodos empregados na mensuração da eficiência no contexto do setor educacional, verifica-se tanto o uso de indicadores da relação insumo e produto, quanto de modelos estatísticos paramétricos e não paramétricos. Uma lista não exaustiva inclui os estudos de Ahn, Charles e Cooper (1988), Breu e Raab (1994), Taylor e Harrys (2004), Glass et al. (2006), França, Figueiredo e Lapa (2010), SellersRubio, Mas-Ruiz e Casado-Díaz (2010), Wolszczak-Derlacz e Parteka (2011), Costa et al. (2012) e Barra e Zotti (2016b).

O método de análise por envoltória de dados (DEA), modelo não paramétrico que relaciona o volume de recursos utilizados com os resultados alcançados, constitui-se na técnica mais empregada na estimação da eficiência de instituições de ensino (COSTA et al., 2012). Sendo o setor educacional composto por múltiplos insumos e múltiplos produtos, a DEA tem sido apontada como a mais apropriada, tendo em vista a possibilidade de inserção de múltiplos fatores de entrada e saída, além de não ser necessário conhecer a forma funcional da função de produção.

Diante disso, o presente estudo baseia-se no seguinte problema de pesquisa: quais os fatores condicionantes da eficiência, no período de 2008 a 2015, das universidades federais brasileiras que aderiram ao Reuni? Buscou-se, portanto, desenvolver uma análise com referência na eficiência relativa das universidades que aderiram ao Reuni, bem como discutir sobre alguns fatores condicionantes dessa eficiência. Sua principal contribuição está na análise do desempenho das universidades durante o período de implantação do Reuni, bem como na aplicação de uma metodologia de avaliação que, além de mensurar a eficiência, busca evidenciar os aspectos que colaboram com a geração de resultados, provendo informações aos gestores, de forma que possam corrigir ou reforçar o desempenho alcançado. Contribui, portanto, com os administradores das universidades; com o governo, ao fornecer um mecanismo que auxilie na avaliação e decisões de investimentos; e com a sociedade em geral, ao apresentar um panorama do desempenho das instituições federais de ensino superior e colaborar com a prestação de contas das políticas públicas.

\section{Eficiência em instituições de ensino superior}

As primeiras contribuições da literatura referentes à análise da eficiência de instituições de ensino superior datam de meados de 1980. Ahn, Charles e Cooper (1988) utilizaram o método Data Envelopment Analysis (DEA) para analisar a eficiência de instituições de nível superior orientadas para a pesquisa nos EUA, 
comparando o desempenho de universidades públicas e privadas, em que as primeiras obtiveram maior nível de eficiência.

A partir de então, diversos foram os estudos realizados com a temática, sendo avaliada a eficiência de universidades em diversos países, utilizando-se, em sua maioria, o método DEA (CostA et al., 2012). Como exemplo, Breu e Raab (1994) mensuraram a eficiência relativa das 25 universidades mais bem ranqueadas nos Estados Unidos, demonstrando que existia uma relação inversa entre o ranking existente e a eficiência mensurada por meio do método DEA.

Ao analisar a eficiência de 21 universidades da África do Sul, no período de 1994 a 1997, Taylor e Harrys (2004) apresentam a discussão de quatro fatores apontados como fundamentais para explicar as diferenças de eficiência entre as universidades. Os maiores números de alunos graduados têm relação positiva com o nível de eficiência, bem como com a qualidade dos alunos ingressantes. A qualificação dos professores e pesquisadores também está associada à maior eficiência. Por outro lado, elevados montantes de custos fixos mostraram-se associados aos menores índices de eficiência.

Glass et al. (2006) mensuraram a eficiência de 98 universidades do Reino Unido e constataram que as universidades mais focadas em uma só atividade (ensino ou pesquisa) eram mais eficientes que aquelas sem especialização, ou seja, aquelas que desenvolvem igualmente tanto atividades de ensino quanto de pesquisa. Observou-se também que as maiores universidades mostraram-se mais eficientes.

França, Figueiredo e Lapa (2010) aplicaram dois modelos a partir do método DEA em 30 universidades federais do Brasil para avaliar o impacto da assimetria de informação sobre a eficiência organizacional, analisando a relação entre agente (universidades) e principal (MEC). Utilizando dados do ano de 1998, a análise das diferenças nos pesos estimados para cada um dos modelos permitiu concluir que existe problema de agência para um grupo de instituições, indicando a necessidade de melhorias em mecanismos de controle.

Em estudo feito com 48 departamentos acadêmicos de uma universidade na Espanha, Sellers-Rubio, Mas-Ruiz e Casado-Díaz (2010) buscaram mensurar a eficiência do ensino e da pesquisa, bem como identificar seus fatores determinantes. A análise empírica mostrou diferentes níveis de eficiência em ensino e eficiência em pesquisa nos departamentos em estudo. A análise dos condicionantes da eficiência permitiu verificar que existe um trade-off entre a eficiência no ensino e a dedicação às atividades de pesquisa. Por outro lado, observou-se complementariedade entre a eficiência no ensino e a eficiência em pesquisa. Os autores defendem que tais resultados não são contraditórios, tendo em vista que a maior eficiência não está associada à maior dedicação (mais horas), mas ao melhor gerenciamento de 
tempo. Observou-se ainda que aqueles departamentos com maior tempo dedicado à pesquisa (aqueles com menor número de créditos de disciplinas) mostraram-se mais eficientes em pesquisa.

Wolszczak-Derlacz e Parteka (2011) analisaram a eficiência e seus condicionantes a partir de uma amostra de 259 instituições públicas de ensino superior da Polônia, Áustria, Finlândia, Alemanha, Itália, Suíça e do Reino Unido. Os resultados indicaram uma considerável variabilidade de escores de eficiência em cada país e entre os países. O tamanho da unidade, representado pelo número total de alunos e número total de faculdades, mostrou-se associado à maior eficiência, sugerindo a existência de economias de escala. As universidades com faculdades de medicina ou farmácia também foram caracterizadas como mais eficientes. A estrutura de gênero da equipe acadêmica também se mostrou importante para o desempenho das instituições, com a presença de mulheres positivamente correlacionada com a eficiência. Outro fator associado aos maiores escores de eficiência foi o tempo de existência das universidades, ou seja, universidades com maior tradição mostraramse mais eficientes que instituições mais novas.

Analisando a eficiência de 49 Instituições Federais de Ensino Superior (IFES) brasileiras, no período de 2004 a 2008, Costa et al. (2012) constataram elevados níveis de eficiência educacional. Entre aquelas universidades com maior atuação em ensino de pós-graduação e pesquisa, as causas da ineficiência são apontadas como o elevado número de alunos em relação ao quantitativo de professores e o aumento do custo por aluno. Por sua vez, nas universidades com pouca ou nenhuma atuação em ensino de pós-graduação e pesquisa, os fatores que mais comprometeram a eficiência foram o elevado número de alunos por professores e alunos por funcionários, bem como o índice de qualificação do corpo docente. 0 impacto negativo desse último indicador sugere, segundo os autores, a existência de muitos professores qualificados e que não estão sendo utilizados em sua plenitude nas IFES, reflexo também da atual política de contratação de docentes, em que se exige maior qualificação dos professores. Constatou-se ainda que as baixas taxas de sucesso na graduação, dadas pela relação entre o número de alunos formados e matriculados, bem como o baixo conceito Capes, afetaram negativamente a eficiência das universidades, independente da dedicação às atividades de pósgraduação e pesquisa.

Barra e Zotti (2016b) mensuraram a eficiência de departamentos e faculdades da Universidade de Salerno, no sul da Itália, analisando separadamente unidades da área de Ciência e Tecnologia e unidades de Humanidades e Ciências Sociais, no período de 2005 a 2009. As análises foram realizadas considerando, separadamente, atividades de ensino e atividades de pesquisa. Os resultados apontaram que os 
departamentos da área de Ciência e Tecnologia mostraram-se mais eficientes em pesquisa que aqueles da área de Humanas e Sociais. Por sua vez, os departamentos da área de Humanidades e Ciências Sociais foram mais eficientes nas atividades de ensino. Os autores salientam ainda que as estimativas de eficiência dependem fortemente da especificação de saída (produto), tendo em vista que a utilização de proxies de qualidade reduziu o desempenho tanto em atividades de ensino quanto em atividades de pesquisa.

Observa-se que, desde os primeiros estudos em que foram empregados métodos não paramétricos para a avaliação do desempenho de instituições de ensino superior, as discussões propõem a análise de características associadas aos níveis de eficiência. No entanto, tais estudos parecem ser ainda incipientes, tendo em vista que seus resultados restringem-se pelos recortes espaço-temporais ou pela limitação das variáveis analisadas. Nesse sentido, outros estudos podem contribuir com a ampliação das discussões acerca dos fatores condicionantes da eficiência em instituições de ensino superior.

\section{Procedimentos metodológicos}

Visando garantir uniformidade e contemporaneidade ao estudo, optou-se por analisar apenas as instituições de ensino superior organizadas academicamente como universidades, cuja categoria administrativa seja pública federal e que tenha aderido ao Reuni.

De acordo com o relatório do primeiro ano do Reuni (BRASIL, 2009), das 54 universidades federais existentes em 2007, 53 aderiram ao programa, com implementação dos planos em 2008. O Reuni estabeleceu metas a serem alcançadas em um período de cinco anos, a contar do início de cada plano. Por isso, o presente estudo abordou o período de 2008 a 2015, compreendendo os dois planos plurianuais do Governo Federal (2008-2011 e 2012-2015) de forma a analisar todo o período do programa em vigor. Em função da disponibilização de dados fora do padrão determinado pelo Tribunal de Contas da União (TCU), excluiuse uma universidade do estudo, que contemplou, portanto, 52 universidades.

O desenvolvimento do trabalho ocorreu por meio dos seguintes procedimentos de investigação: a) geração de escores de eficiência, pelo uso da análise por envoltória de dados (DEA) e b) identificação dos fatores associados à eficiência, utilizando-se a análise de regressão Tobit, que são explicitados a seguir. 


\section{Análise por envoltória de dados (DEA)}

A análise por envoltória de dados (DEA - Data Envelopment Analysis) é um método que teve origem no trabalho pioneiro de Farrell (1957), sendo posteriormente desenvolvido por Charnes, Cooper e Rhodes (1978) e avalia a eficiência relativa no uso dos recursos de empresas pertencentes a um mesmo setor ou ramo de atividades.

De acordo com Soares de Mello et al. (2001), na versão inicial da DEA, as unidades analisadas eram supostamente capazes de tomar decisões sobre o nível de insumos utilizados e sobre a quantidade de produtos resultantes, sendo consideradas unidades tomadoras de decisão, ou DMU (Decision Making Units). Os autores salientam ainda que a grande vantagem da DEA é permitir que cada unidade avaliada busque a eficiência de maneira distinta, de tal forma que atenda às suas especificidades.

A DEA apresenta dois modelos básicos de análise: o modelo com retornos constantes, ou modelo CCR, desenvolvido por Charnes, Cooper e Rhodes; e o modelo BCC, também conhecido por modelo com retornos variáveis, que foi desenvolvido por Banker, Charnes e Cooper. Tanto o modelo CCR quanto o BCC podem ser analisados pela ótica da orientação para o consumo ou pela orientação para o produto. A escolha da orientação não exerce grande influência na magnitude do valor de eficiência técnica, e o critério de escolha dependerá da finalidade do estudo, ou seja, se o objetivo é regularizar o consumo de insumos ou possibilitar o aumento da produção. (GOMES; BAPTISTA, 2004).

Considerando as características dessas unidades, em relação ao porte, ao montante de recursos utilizados e à variação dos resultados divulgados conforme a coleta de dados, o modelo com retornos variáveis se mostra mais apropriado. Quanto à orientação do modelo, sendo as universidades federais brasileiras unidades vinculadas à administração pública, percebe-se que a natureza dos insumos utilizados, tais como número de docentes, recursos financeiros, já possui caráter de escassez na oferta, além de que essas unidades não têm muito poder de decisão sobre o nível de insumos. Nesse sentido, o modelo que busca a eficiência voltada para a maximização da produção apresenta-se mais condizente com a natureza das atividades dessas organizações. A escolha desse modelo corrobora a opção metodológica adotada por Belloni (2000), Eckles (2010), Obadic e Aristovnik (2011), Barra e Zotti (2016a) e Barra e Zotti (2016b).

Para Soares de Mello et al. (2005), o método DEA com orientação-produto e que pressupõe retornos variáveis de escala (BCC) é modelado em (1), em que $x_{i k}$ representa o input $i$ da $\mathrm{DMU}_{k} ; y_{j k}$ representa o output j da $\mathrm{DMU}_{k} ; \lambda_{k}$ é a contribuição da $\mathrm{DMU}^{k}$ na formação do alvo da $\mathrm{DMU}_{0} \mathrm{e} h_{0}$ é a eficiência. 


$$
\begin{aligned}
& \operatorname{Max}_{0} \\
& \text { sujeito a } \\
& x_{i 0}-\sum_{k=1}^{n} \mid x_{i k} \lambda_{k} \geq 0, \forall i \\
& -h_{0} y_{j 0}+\sum_{k=1}^{n} y_{j k} \lambda_{k} \geq 0, \forall j \\
& \sum_{k=1}^{n} \lambda_{k}=1 \\
& \lambda_{k} \geq 0, \forall k
\end{aligned}
$$

Buscou-se incorporar no presente estudo variáveis que expressem os resultados das atividades desenvolvidas, tanto em termos do quantitativo de alunos atendidos, quanto da qualidade dos cursos oferecidos pelas universidades, conforme mencionadas a seguir:

- Variável de insumo $(x)$

i) CustoC: custo corrente da universidade, valor calculado conforme orientações do TCU (BRASIL, 2002), é representado pelo total das despesas correntes, menos 65\% das despesas correntes dos hospitais universitários, deduzindo-se ainda despesas com aposentadorias e reformas, pensões, sentenças judiciais, despesas com pessoal cedido ou com afastamento (docentes e técnicos administrativos).

- Variáveis de produto (y)

ii) AluGra: alunos de graduação, resultado do produto do número de alunos equivalentes na graduação $\left(A_{G} E\right)$ pelo Conceito Médio da Graduação (CMG) da universidade (média do conceito preliminar (CPC) de cada curso, indicador de qualidade que avalia os cursos de graduação com base na avaliação de desempenho de estudantes, no valor agregado pelo processo formativo e em insumos referentes às condições de oferta, como o corpo docente, infraestrutura e recursos didático-pedagógicos). O $A_{G} E$ é um indicador divulgado no relatório de gestão das universidades federais e, conforme determinações do TCU (BRASIL, 2002), é expresso em (2):

$A_{G} E=\sum$ todos os cursos $\left\{\left(N_{D I} \times D_{P C}\right)(1+\right.$ Fator de Retenção $)+\left(\left(N_{I}-N_{D I}\right) / 4 \times\right.$ $\left.\left.D_{P C}\right)\right\} \times$ Peso do grupo em que se insere o curso

em que $N_{D I}$ é o número de diplomados no ano, $D_{P C}$ expressa a duração padrão do curso, $N_{I}$ é o número de alunos ingressantes no ano, e Fator de Retenção e Peso do grupo são calculados de acordo com metodologia da Secretaria de Educação Superior (SESu). 
iii) AluPos: alunos de pós-graduação, composto pelo produto do número total de alunos regularmente matriculados em cursos de mestrado e doutorado (excluindose as matrículas de alunos dos cursos de mestrado profissionalizante, conforme orientações do TCU (BRASIL, 2002)) e a média do conceito Capes de todos os programas de pós-graduação oferecidos pela universidade.

\section{Análise de regressão Tobit}

Para a identificação dos fatores condicionantes da eficiência das universidades federais brasileiras utilizou-se o modelo econométrico Tobit. Vários estudos que empregaram o modelo DEA para mensurar a eficiência de determinadas unidades utilizaram também o modelo Tobit, combinando o modelo econométrico para identificar fatores determinantes da eficiência. Uma lista não exaustiva inclui os trabalhos de Kirjavainen e Loikkanentna (1998) na mensuração da eficiência na área do ensino secundário, Sellers-Rubio, Mas-Ruiz e Casado-Díaz (2010) e Agasisti (2011), que analisaram a eficiência do ensino superior e Moreira et al. (2011), na análise da pós-graduação.

Segundo Wooldridge (2013), a utilização do Tobit é apropriada nos casos em que a variável dependente limitada é uma resposta de solução de canto. Esse tipo de variável distribui-se de forma concentrada em determinados pontos da amostra, mas é aproximadamente distribuída de forma contínua com valores acima ou abaixo desse ponto.

No presente estudo, o modelo Tobit apresenta-se apropriado uma vez que, conforme análise exploratória dos dados, a variável dependente mostra-se concentrada em 1. Segundo Greene (1997), o modelo Tobit pode ser definido da seguinte forma:

$$
y_{i}^{*}=\beta^{\prime} x_{i}+\varepsilon_{i}
$$

em que $\varepsilon_{i}$ é o erro aleatório da equação; $\beta$ é o vetor de parâmetros e representa os coeficientes a serem estimados; $x_{i}$ representa as variáveis explicativas; e, $y_{i}^{*}$ é a variável dependente estimada.

A variável $y_{i}$ que é efetivamente observada, qual seja o escore de eficiência gerado, é definida da seguinte forma:

$$
\left\{\begin{array}{l}
y_{i}=y_{i}^{*} \text { se } y_{i}^{*}<y_{i}^{c} \\
y_{i}=y_{i}^{c} \text { se } y_{i}^{*} \geq y_{i}^{c}
\end{array}\right.
$$


Na equação (4), $y_{i}^{c}$ representa o valor da censura e, de acordo com os propósitos do presente trabalho, o valor da censura é 1 . Dessa forma, tem-se a seguinte formulação:

$$
\left\{\begin{array}{l}
y_{i}=y_{i}^{*} \text { se } y_{i}^{*}<1 \\
y_{i}=1 \text { se } y_{i}^{*} \geq 1
\end{array}\right.
$$

Para estimar a regressão em que a amostra é censurada não se pode utilizar o Método dos Mínimos Quadrados Ordinários, uma vez que os parâmetros extraídos seriam tendenciosos e inconsistentes, pela ausência de informações de algumas observações, ou pela censura dos dados observados. Logo, as estimativas do modelo Tobit, ou de regressão censurada, são obtidas por meio do Método da Máxima Verossimilhança. (GUJARATI, 2000)

Dessa forma, segundo Greene (1997), o valor esperado de $y_{i}$, dadas as variáveis independentes $x_{i}\left(x_{1}, x_{2} \ldots x_{n}\right)$, é encontrado por meio da equação (6):

$$
\begin{aligned}
& E\left(y_{i} \mid x_{i}\right)=\operatorname{Prob}\left(y_{i}=1\right) \times E\left(y_{i} \mid y_{i}=1\right)+\operatorname{Prob}\left(y_{i}<1\right) \times E\left(y_{i} \mid y_{i}<1\right) \\
& =x_{i}^{\prime} \beta \Phi\left(\frac{\beta x_{i}^{\prime}}{\sigma}\right)+\sigma \phi\left(\frac{\beta x_{i}^{\prime}}{\sigma}\right)
\end{aligned}
$$

em que $\sigma$ representa o desvio padrão dos termos de erro; $\Phi$ e $\phi$ representam, respectivamente, a função distribuição cumulativa normal e a função densidade normal avaliadas em $\beta x_{i}^{\prime} / \sigma$.

Vale destacar que, no presente estudo, foi utilizado o modelo Tobit com dados em painel. Os modelos estatísticos com dados em painel são apropriados para combinar dados sobre distintas unidades de análise coletados em diferentes períodos de tempo, como dias, semanas, meses, anos, entre outros. Apresentam vantagens em relação aos modelos de série temporal e de corte seccional (cross secction). Dentre elas destaca-se a redução do problema de colinearidade entre as variáveis explicativas, uma vez que os modelos com dados em painel permitem um maior número de observações na análise, aumentando os graus de liberdade e a eficiência dos parâmetros estimados.

Tendo em vista que a estimação da regressão Tobit é feita por máxima verossimilhança, uma limitação é que os modelos em painel devem ser estimados por efeitos aleatórios, já que os efeitos fixos apresentam o problema de parâmetros incidentais. (WOOLDRIDGE, 2013) 
- Variável dependente (y)

É constituída pelo escore de eficiência gerado por meio da análise por envoltória de dados. Dessa forma, cada universidade tem um coeficiente positivo que varia de 0 a 1, calculado por meio do modelo BCC com orientação para o produto.

- Variáveis independentes $(x)$

O conjunto das variáveis explicativas incluídas no presente estudo procura abordar aspectos considerados no relatório de gestão, bem como propor uma reflexão sobre a influência de alguns fatores na eficiência das universidades federais brasileiras. Dessa forma, os fatores analisados e as variáveis incluídas no modelo econométrico Tobit são descritos a seguir.

i) AluPro: representa a composição do corpo docente utilizado no período e é resultado da divisão do total de alunos em tempo integral (indicador calculado conforme determinações do TCU e representa o número de alunos de graduação, considerando ingressantes, diplomados, duração padrão do curso e fator de retenção, acrescido do total de alunos de pós-graduação e residência, sendo atribuído peso 2 nos dois últimos) pelo total de professores equivalentes (somatório do número de professores ponderado pelo regime de trabalho, sendo peso 0,5 para o regime de 20 horas semanais e peso 1,0 para os regimes de 40 horas e dedicação exclusiva, excluindo-se aqueles afastados para capacitação ou cedidos para outros órgãos).

ii) AluFun: expressa a composição do pessoal técnico e é descrita pelo resultado da divisão do total de alunos em tempo integral pelo total de funcionários equivalentes (somatório do número de funcionários técnico-administrativos vinculados à universidade ou contratados sob a forma de temporários, ponderado pelo regime de trabalho, sendo peso 0,5 para o regime de 20 horas semanais, 0,75 para 30 horas e 1,0 para 40 horas semanais, excluindo-se aqueles afastados para capacitação ou cedidos para outros órgãos).

iii) FunPro: representa o suporte fornecido pelo pessoal de nível técnico às atividades desenvolvidas pelos docentes e resulta da divisão do número de funcionários equivalentes pelo número de professores equivalentes.

iv) QuaDoc: é o índice de qualificação do corpo docente e consiste na divisão da soma ponderada dos professores (somatório do número de professores em exercício efetivo, mais substitutos e visitantes, ponderado pela qualificação, considerando apenas o maior título, sendo atribuído peso 5 aos docentes doutores, 3 aos mestres, 2 aos docentes com especialização e 1 aos docentes graduados) pelo total de professores. Não se considera nesse cálculo o regime de trabalho dos docentes, e excluem-se aqueles docentes afastados para capacitação ou cedidos para outros órgãos. 
v) EnvPos: demonstra o grau de envolvimento com atividades de pós-graduação, sendo composto pela relação entre o número de alunos matriculados na pósgraduação e o total de alunos da universidade (graduação mais pós-graduação).

vi) TemExi: representa o tempo de existência da universidade desde a sua federalização;

vii) SerTot: o número total de servidores, utilizado como proxy do tamanho da universidade, é composto pela soma dos funcionários equivalentes e professores equivalentes, que incluem servidores vinculados à universidade, bem como os contratados.

viii) CamFor: é o número de campi instalados em municípios fora da sede administrativa da universidade.

\section{Resultados}

Buscou-se mensurar a eficiência das universidades federais brasileiras, bem como levantar discussões acerca de seus fatores condicionantes, no período de 2008 a 2015, conforme os procedimentos metodológicos delineados. Portanto, nos tópicos a seguir são expostos os principais resultados da pesquisa.

\section{Indicadores de eficiência das universidades federais brasileiras}

As estatísticas descritivas das variáveis utilizadas na mensuração da eficiência das universidades são apresentadas nas Tabelas 1 e 2. Pode-se observar que o custo corrente (CustoC) das universidades federais (Tabela 1) mostra grande dispersão em torno da média, caracterizando a heterogeneidade dessas instituições em termos da utilização de recursos financeiros para o financiamento de suas atividades. Essa variação, apesar de bastante expressiva, apresenta decréscimo ao longo do período analisado, conforme denota o coeficiente de variação. 
Tabela 1 - Estatísticas descritivas da variável de insumo custo corrente, Brasil, 2008-2015

\begin{tabular}{ccccccc}
\hline CustoC & Média & Desvio padrão & $\begin{array}{c}\text { Coeficiente } \\
\text { de variação }\end{array}$ & Máximo & Mínimo \\
\hline 2008 & $252.862 .502,28$ & $196.086 .396,50$ & $77,55 \%$ & $796.027 .365,57$ & $28.423 .667,00$ \\
\hline 2009 & $285.436 .469,98$ & $223.769 .953,94$ & $78,40 \%$ & $957.417 .333,18$ & $37.135 .182,08$ \\
\hline 2010 & $341.270 .003,20$ & $261.347 .435,65$ & $76,58 \%$ & $1.163 .609 .904,68$ & $42.842 .370,15$ \\
\hline 2011 & $379.913 .074,93$ & $284.792 .129,04$ & $74,96 \%$ & $1.291 .735 .982,04$ & $53.610 .035,44$ \\
\hline 2012 & $402.987 .159,42$ & $292.815 .433,95$ & $72,66 \%$ & $1.207 .588 .375,61$ & $62.284 .841,71$ \\
\hline 2013 & $487.562 .973,81$ & $356.362 .744,64$ & $73,09 \%$ & $1.663 .752 .853,25$ & $72.303 .277,00$ \\
\hline 2014 & $530.723 .934,42$ & $381.185 .985,19$ & $71,82 \%$ & $1.900 .792 .747,67$ & $84.353 .006,57$ \\
\hline 2015 & $559.825 .272,50$ & $384.537 .561,45$ & $68,69 \%$ & $2.056 .586 .531,31$ & $115.252 .428,10$ \\
\hline
\end{tabular}

Fonte: resultados da pesquisa (2018).

As diferentes demandas de recursos, em função da área do conhecimento dos cursos ofertados em cada instituição, bem como o próprio tamanho da estrutura e a quantidade de cursos oferecidos em cada universidade são fatores que podem justificar essa grande dispersão em torno dos valores médios do custo corrente.

$\mathrm{Na}$ Tabela 2 são apresentadas as estatísticas descritivas das variáveis que representam os produtos gerados pelas instituições de ensino superior: i) alunos de graduação (AluGra) e ii) alunos de pós-graduação (AluPos). Ressalta-se que a variável AluGra é resultado do produto do número de alunos equivalentes de graduação pelo conceito médio da graduação (CMG) da universidade, indicador que compõe o índice geral de cursos (IGC). Em função da não disponibilidade de dados para o cômputo dessa variável em 2008, excepcionalmente, utilizou-se o IGC em substituição ao CMG. Além disso, também em 2008, uma universidade não teve o CMG nem o IGC divulgados, não sendo considerada na análise desse ano. Por sua vez, a variável AluPos resulta do produto do número de alunos matriculados em cursos de pós-graduação stricto sensu pela média do Conceito Capes da instituição. 
Tabela 2 - Estatísticas descritivas das variáveis de produto alunos de graduação e alunos de pós-graduação, Brasil, 2008-2015

\begin{tabular}{cccccc}
\hline AluGra & Média & Desvio padrão & $\begin{array}{c}\text { Coeficiente de } \\
\text { variação }\end{array}$ & Máximo & Mínimo \\
\hline 2008 & $55.349,50$ & $40.984,40$ & $74,05 \%$ & $180.013,00$ & $4.656,80$ \\
\hline 2009 & $52.560,90$ & $38.625,00$ & $73,49 \%$ & $178.422,30$ & $4.465,90$ \\
\hline 2010 & $57.577,40$ & $40.092,50$ & $69,63 \%$ & $187.127,70$ & $7.165,70$ \\
\hline 2011 & $59.987,31$ & $37.338,48$ & $62,24 \%$ & $169.736,80$ & $8.829,80$ \\
\hline 2012 & $60.834,30$ & $39.204,10$ & $64,44 \%$ & $182.734,70$ & $8.883,60$ \\
\hline 2013 & $61.367,00$ & $36.262,40$ & $59,09 \%$ & $154.359,60$ & $9.497,10$ \\
\hline 2014 & $64.493,30$ & $36.879,10$ & $57,18 \%$ & $170.850,70$ & $7.804,80$ \\
\hline 2015 & $66.750,40$ & $38.021,80$ & $56,96 \%$ & $158.415,90$ & $14.935,00$ \\
\hline AluPos & & & & & \\
\hline 2008 & $8.657,70$ & $11.272,30$ & $130,20 \%$ & $46.927,10$ & 117,00 \\
\hline 2009 & $8.935,70$ & $11.588,10$ & $129,68 \%$ & $48.714,00$ & 141,00 \\
\hline 2010 & $10.010,80$ & $12.466,50$ & $124,53 \%$ & $52.639,50$ & 150,00 \\
\hline 2011 & $10.795,10$ & $13.143,20$ & $121,75 \%$ & $52.377,60$ & 219,00 \\
\hline 2012 & $11.176,30$ & $13.659,90$ & $122,22 \%$ & $55.271,60$ & 339,00 \\
\hline 2013 & $12.086,90$ & $14.529,10$ & $120,21 \%$ & $57.932,60$ & 609,00 \\
\hline 2014 & $13.213,40$ & $15.214,90$ & $115,15 \%$ & $60.877,40$ & 561,00 \\
\hline 2015 & $13.709,50$ & $15.695,70$ & $114,49 \%$ & $61.607,60$ & 489,00 \\
\hline
\end{tabular}

Fonte: resultados da pesquisa (2018).

Nota-se que o quantitativo de alunos também mostrou ampla variação em torno da média, reforçando as considerações acerca da diferença de magnitude entre as unidades analisadas. É notável a expressiva variabilidade do número de alunos da pós-graduação (AluPos), denotando existir grandes diferenças na dedicação às atividades de ensino da pós-graduação.

Assim como na variável custo corrente (CustoC), nota-se que, ao longo do período analisado, as variáveis AluGra e AluPos têm apresentado diminuição do coeficiente de variação e, apesar de ainda expressarem uma ampla variabilidade, indicam que, com o passar do tempo, as universidades estão se tornando mais homogêneas.

As estatísticas descritivas dos escores de eficiência são apresentadas na Tabela 3. Em média, nota-se que as universidades federais operam aquém de suas capacidades produtivas, tendo em vista que apresentaram resultados abaixo do potencial, se comparados aos melhores desempenhos observados em cada ano. 
Tabela 3 - Estatísticas descritivas dos escores de eficiência das universidades federais, Brasil, 2008-2015

\begin{tabular}{cccccc}
\hline Eficiência & Média & $\begin{array}{c}\text { Desvio } \\
\text { padrão }\end{array}$ & $\begin{array}{c}\text { Coeficiente } \\
\text { de variação }\end{array}$ & Máximo & Mínimo \\
\hline 2008 & 0,7731 & 0,1898 & $24,55 \%$ & 1,0000 & 0,2500 \\
\hline 2009 & 0,6902 & 0,2001 & $28,99 \%$ & 1,0000 & 0,2092 \\
\hline 2010 & 0,7178 & 0,1795 & $25,01 \%$ & 1,0000 & 0,3375 \\
\hline 2011 & 0,7376 & 0,1838 & $24,92 \%$ & 1,0000 & 0,2810 \\
\hline 2012 & 0,7672 & 0,1580 & $20,60 \%$ & 1,0000 & 0,4230 \\
\hline 2013 & 0,7585 & 0,1683 & $22,18 \%$ & 1,0000 & 0,4031 \\
\hline 2014 & 0,7546 & 0,1784 & $23,63 \%$ & 1,0000 & 0,3191 \\
\hline 2015 & 0,7544 & 0,1866 & $24,73 \%$ & 1,0000 & 0,4156 \\
\hline
\end{tabular}

Fonte: resultados da pesquisa (2018).

Ao longo do período analisado, é possível notar uma redução na eficiência média, especialmente no ano de 2009, em que as universidades que aderiram ao Reuni atingiram o menor escore de eficiência e mostraram-se com desempenhos mais heterogêneos. Nos anos seguintes, a evolução do desempenho médio demonstrou que as universidades apresentaram, comparativamente, melhoria na utilização de recursos, alcançando, em 2012, nível de eficiência bem próximo ao observado em 2008 e com menor dispersão dos desempenhos médios. Nos demais anos, os indicadores apresentaram pequena diminuição da eficiência, fechando em 2015 com escores ligeiramente abaixo daqueles observados no ano de implantação do Reuni.

Vale ressaltar que, por se tratar de uma medida relativa, os escores de eficiência mensurados em determinado ano servem como referência do desempenho médio daquele período, tendo em vista que os melhores resultados observados foram apontados como a máxima eficiência, sendo os demais escores calculados em termos comparativos. No entanto, não se pode afirmar que os melhores resultados observados sejam de fato a maior eficiência possível naquele ano, e, dessa forma, as oscilações nos valores médios dos escores de um ano para outro refletem muito mais a dispersão entre os desempenhos do que reais ganhos ou perdas de eficiência nas universidades. Nesse sentido, a comparação do nível de eficiência entre os anos deve ser considerada apenas como uma medida de dispersão do desempenho, em que as menores médias indicam grande heterogeneidade de eficiência no respectivo período. 


\section{Condicionantes da eficiência das universidades federais brasileiras}

Para a análise dos fatores associados à eficiência das universidades, procedeuse à estimação da regressão Tobit, considerando dados em painel com efeitos aleatórios, tendo como variável dependente o escore de eficiência gerado por meio do método DEA orientado para o produto e que pressupõe retornos variáveis de escala (DEA-BCC). As estatísticas descritivas das variáveis independentes, quais sejam: i) relação alunos por professor (AluPro), ii) relação alunos por funcionário (AluFun), iii) relação funcionários por professor (FunPro), iv) qualificação do corpo docente (QuaDoc), v) envolvimento com atividades de pós-graduação (EnvPos), vi) o tempo de existência da universidade (TemExi), vii) total de servidores (SerTot) e viii) o número de campi fora da sede (CamFor), são apresentadas na Tabela 4.

Tabela 4 - Estatísticas descritivas das variáveis independentes utilizadas na análise da eficiência das universidades federais, Brasil, 2008-2015

\begin{tabular}{|c|c|c|c|c|c|c|c|c|c|}
\hline & & & & 0 & $\mathrm{C}$ & s & $\mathbf{x i}$ & t & \\
\hline \multirow{8}{*}{ 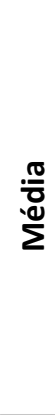 } & 2008 & & & & & & & & \\
\hline & 2 & & & & & & & & \\
\hline & 200 & & & & & & & & \\
\hline & 2011 & 35 & 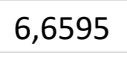 & 4 & 2 & 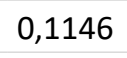 & 2 & & 38 \\
\hline & 2012 & 11,8465 & 9 & & & & & & 23 \\
\hline & 2013 & & & & & & & & \\
\hline & 20 & - & & & & & & & \\
\hline & 20 & & & & & & & & \\
\hline \multirow{8}{*}{ 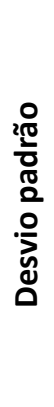 } & 2008 & & & & & & & & \\
\hline & 200 & 2,7 & & & & & & & \\
\hline & 01 & r & & & & & & & \\
\hline & 011 & 年 & 2,00 & & & & & & \\
\hline & 012 & 2,7197 & & & & & & & \\
\hline & 201 & r & 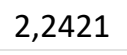 & & & 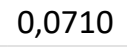 & 16 & & \\
\hline & 20 & 2,3150 & & & & & & & \\
\hline & 2 & 2,2445 & 2,2264 & 0,5900 & 0,3858 & 0,0693 & 16,7609 & $3.272,27$ & 2,4163 \\
\hline
\end{tabular}




\begin{tabular}{|c|c|c|c|c|c|c|c|c|c|}
\hline & & ro & AluFun & FunPro & QuaDoc & EnvPos & TemExi & SerTot & CamFor \\
\hline \multirow{8}{*}{ 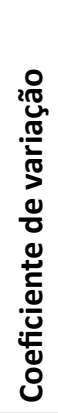 } & 2008 & 6 & $\%$ & $\%$ & $\%$ & $\%$ & $\%$ & $5,52 \%$ & $35,07 \%$ \\
\hline & 2009 & (4) & $32,72 \%$ & $42,79 \%$ & 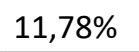 & $70,09 \%$ & $40,13 \%$ & $54 \%$ & ,07\% \\
\hline & 2010 & 1 & $10 \%$ & 3 & 1 & $\%$ & $\%$ & $\%$ & $\%$ \\
\hline & 2011 & 22 & $\%$ & 3 & $10,68 \%$ & & $\%$ & & $3 \%$ \\
\hline & 2012 & $22,96 \%$ & 32 & 3 & $9,54 \%$ & $29 \%$ & $\%$ & $\%$ & $\%$ \\
\hline & 2013 & $21,30 \%$ & 34 & 38 & $10,02 \%$ & $40 \%$ & $36,62 \%$ & $\%$ & 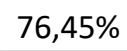 \\
\hline & 2014 & -01 & - & $\%$ & 10 & $\%$ & $\%$ & & \\
\hline & 2015 & $17,51 \%$ & 70 & $8 \%$ & 9,0 & $\%$ & $9 \%$ & & \\
\hline \multirow{8}{*}{ 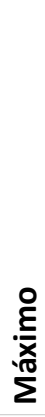 } & 2008 & 18,7100 & 18,3300 & 7,6300 & 4,8390 & 60 & 0000 & 6,00 & 9,0000 \\
\hline & 2009 & 16,5200 & 14,1800 & 5,6000 & 4,8700 & 0,3840 & 89,0000 & $11.790,00$ & 10,0000 \\
\hline & 2010 & 16,8900 & 00 & 00 & 4,8 & 40 & 9 & 00 & 10 \\
\hline & 2011 & 10, & 0 & 4,4400 & 5 , & & & & \\
\hline & 2012 & 16 & 0 & 4,1900 & 5 & 0,3000 & & 0 & \\
\hline & 2013 & 16,7800 & 00 & Do & 5 & oo & 0 & , 00 & 0 \\
\hline & 2014 & 16,1400 & 13,4000 & 3,3600 & 5,2400 & 0,3000 & 0000 & $18.551,00$ & 10,0000 \\
\hline & 2015 & 16,6100 & 14,3700 & 3,7100 & 5,2100 & 0,3000 & 95,0000 & $18.770,00$ & 10,0000 \\
\hline \multirow{8}{*}{ : $\stackrel{\stackrel{\circ}{\underline{S}}}{\text { : }}$} & 2008 & 1,1000 & העם & 0,0000 & 2,8 & , & 0,0 & (2) & 0,0 \\
\hline & 2009 & 5,1600 & 19000 & ค & 2,8300 & 0013 & & & \\
\hline & 2010 & 7,5000 & 2,24 & 0 & 3, & 6 & 000 & & 0 \\
\hline & 2011 & 3,7600 & 1,9400 & 0,7500 & 2,9400 & 0,0162 & 00 & 30 & 00 \\
\hline & 2012 & 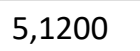 & 0 & 91 & 00 & 100 & 00 & 80 & 0,0000 \\
\hline & 2013 & ,4500 & 2,1300 & 0,7900 & 3,1800 & 0200 & 8,0000 & 603,80 & 0,0000 \\
\hline & 2014 & 7,3400 & 9800 & 0,9900 & 3,2800 & 0200 & 9,0000 & 624,55 & 0,0000 \\
\hline & 2015 & 7,4100 & 3,5600 & 1,0400 & 3,3600 & 0,0200 & 10,0000 & 659,05 & 0,0000 \\
\hline
\end{tabular}

Fonte: resultados da pesquisa (2018).

Verifica-se que o número médio de alunos por professor (AluPro) sofreu pequeno decréscimo nos anos de 2009 e 2010, voltando a crescer nos anos seguintes até alcançar valor ligeiramente superior ao observado em 2008. Importante destacar que, apesar dos valores médios não apresentarem mudanças significativas ao longo do tempo, a dispersão desses dados em torno da média mostra que as universidades tornaram-se consideravelmente mais homogêneas. Nota-se que aquelas instituições que apresentavam número reduzido de alunos por professor, ao longo do tempo, foram ampliando o quantitativo de matrículas.

Evolução semelhante é observada na relação entre o número de alunos por funcionário (AluFun), apresentando também ligeiro aumento desse indicador. No entanto, ressalta-se que a variabilidade dessa variável é significativamente maior, como mostra o coeficiente de variação. Portanto, em termos da composição do 
corpo técnico e administrativo das universidades, existem grandes diferenças entre elas, como se pôde verificar também pela relação entre o número de funcionário e professores (FunPro), que apresentou comportamento semelhante no período analisado.

A média do índice de qualificação do corpo docente (QuaDoc) apresentou crescimento constante de 2008 a 2015, sugerindo existir uma preocupação por parte da administração das universidades em incentivar a capacitação de seus docentes, bem como priorizar a contratação de professores com os mais elevados títulos acadêmicos. A variação desse índice em torno da média tem diminuído nos últimos anos, embora os valores mínimos ainda apontem universidades com baixa titulação média dos docentes.

Em média, o envolvimento das universidades com atividades de pós-graduação (EnvPos) reduziu levemente no período de 2009 a 2013, sugerindo que o quantitativo de alunos de graduação cresceu mais que as matrículas em cursos de pós-graduação nesse período. Nos anos de 2014 e 2015 esse indicador voltou a crescer, superando um pouco o valor alcançado em 2008. No entanto, a variabilidade desse aspecto reduziu consideravelmente, e os valores máximos e mínimos observados sugerem que aquelas instituições com maior dedicação às atividades de pós-graduação acabaram ampliando a oferta de vagas em cursos de graduação, assim como as universidades com pequeno envolvimento com a pós-graduação passaram a oferecer vagas nesse nível de ensino, tornando-as um pouco mais homogêneas.

Com relação ao tempo de existência (TemExi), no início do período analisado, a universidade mais antiga funcionava há 88 anos, enquanto a mais nova havia sido federalizada há apenas 3 anos. Ressalta-se que, dada a dificuldade de apontar com clareza a data de início das atividades de algumas universidades, tendo em vista que muitas delas iniciaram como escolas ou faculdades isoladas, por meio de iniciativas privadas, ou mesmo de governos municipais ou estaduais, adotou-se a data de sua federalização como marco de sua criação.

O total de servidores (SerTot) foi utilizado como proxy do tamanho das universidades, e suas estatísticas descritivas apontam que, apesar do coeficiente de variação mostrar-se em decréscimo, ainda existe expressiva heterogeneidade entre as instituições. Pôde-se verificar também que, em média, os quadros de servidores técnicos e professores apresentaram crescimento ao longo do período analisado, possivelmente para atender à expansão das matrículas no ensino superior.

Expressiva diferença entre as universidades federais também é observada quando se compara o número de campi fora da sede (CamFor). Apesar da média crescente no número de campi de 2008 a 2015, algumas universidades não aderiram à proposta de interiorização do ensino superior, optando por expandir-se 
internamente, e não dispõem de campus instalado fora da cidade sede, enquanto outras instituições adotaram a política de expansão tornando-se multicampi, chegando a abrigar unidades em até 10 municípios diferentes.

Ressalta-se que, pelo fato da variável SerTot não ser representada por índice e apresentar valores de magnitude superior às demais, na análise de regressão (Tabela 5) utilizou-se o In dessa variável, uma vez que a utilização dos valores brutos poderia comprometer o ajustamento do modelo.

Tabela 5 - Fatores associados à eficiência das universidades federais, Brasil, 2008-2015

\begin{tabular}{ccccccc}
\hline Variável & Coeficiente & $\begin{array}{c}\text { Erro } \\
\text { padrão }\end{array}$ & $\mathbf{Z}$ & $\mathbf{P}>|\mathbf{z}|$ & $\mathbf{9 5 \%}$ Intervalo de conf. \\
\hline AluPro & 0,035850 & 0,004179 & 8,58 & $0,0000^{* *}$ & 0,027659 & 0,044041 \\
\hline AluFun & 0,018676 & 0,005446 & 3,43 & $0,0010^{* *}$ & 0,008003 & 0,029349 \\
\hline FunPro & $-0,027245$ & 0,018740 & $-1,45$ & 0,1460 & $-0,063974$ & 0,009484 \\
\hline QuaDoc & $-0,034739$ & 0,029716 & $-1,17$ & 0,2420 & $-0,092981$ & 0,023503 \\
\hline EnvPos & $-0,053189$ & 0,236077 & $-0,23$ & 0,8220 & $-0,515892$ & 0,409513 \\
\hline TemExi & 0,001254 & 0,001570 & 0,80 & 0,4240 & $-0,001822$ & 0,004330 \\
\hline SerTot & 0,067294 & 0,029943 & 2,25 & $0,0250 *$ & 0,008607 & 0,125981 \\
\hline CamFor & $-0,019430$ & 0,007587 & $-2,56$ & $0,0100^{* *}$ & $-0,034300$ & $-0,004561$ \\
\hline Cons & $-0,119075$ & 0,209806 & $-0,57$ & 0,5700 & $-0,530286$ & 0,292137 \\
\hline sigma_u & 0,146445 & 0,017907 & 8,18 & 0,0000 & 0,111347 & 0,181543 \\
\hline sigma_e & 0,097800 & 0,004053 & 24,13 & 0,0000 & 0,089857 & 0,105743 \\
\hline Rho & 0,691564 & 0,055497 & & & 0,575851 & 0,790825
\end{tabular}

Variável dependente: escores de eficiência das universidades federais brasileiras, mensurados por meio da DEABCC com orientação para o produto.

Variáveis independentes: AluPro, relação entre o número de alunos em tempo integral e o número de professores equivalentes; AluFun, relação entre o número de alunos em tempo integral e o número de funcionários equivalentes; FunPro, relação entre o número de funcionários equivalentes e o número de professores equivalentes; QuaDoc, Índice de Qualificação do Corpo Docente; EnvPos, relação entre o número de alunos matriculados na pós-graduação e o total de alunos da universidade; TemExi, tempo de existência da universidade; SerTot, soma do número de funcionários equivalentes e professores equivalentes; e CamFor, número de campi fora da sede.

* significativo a $5 \%$

** significativo a $1 \%$

Fonte: resultados da pesquisa (2018).

Os coeficientes estimados por meio do modelo apresentaram ajustamento satisfatório, demonstrando que as variáveis incluídas no estudo têm considerável 
poder de explicação para a eficiência, conforme verificado pelo teste $x^{2}$, que se apresentou significativo a $1 \%$.

Pode-se perceber que apenas quatro variáveis incluídas no modelo mostraramse significativas para explicar a eficiência das universidades federais, isto é AluPro, AluFun, SerTot e CamFor. Portanto, não se pôde identificar a influência no nível de eficiência da relação funcionários por professor (FunPro), da qualificação do corpo docente (QuaDoc), nem do envolvimento com atividades da pós-graduação (EnvPos), tampouco do tempo de existência (TemExi) das universidades, tendo em vista que tais variáveis não se apresentaram estatisticamente significativas.

Constatou-se uma relação positiva entre o número de alunos por professor (AluPro) e os escores de eficiência, ou seja, aquelas instituições cujo corpo docente atende ao maior quantitativo de alunos mostraram-se mais eficientes na utilização de recursos. Relação positiva também foi identificada entre o número de alunos por funcionários (AluFun) e a eficiência. Essas relações podem ser explicadas pelo fato do acréscimo no número de alunos matriculados não afetar os custos na mesma proporção, impactando positivamente o escore de eficiência.

Analisando o tamanho das universidades, pôde-se perceber que a variável SerTot mostrou-se positivamente associada com a eficiência, indicando que a dimensão das instituições influencia o seu nível de desempenho. Dessa forma, as maiores instituições, identificadas pela magnitude do quadro de servidores técnicos e docentes, mostraram-se mais eficientes.

Outro aspecto analisado foi a existência de multicampi e seu impacto no escore de eficiência. Observou-se que o número de campi fora da sede (CamFor) está negativamente associado à eficiência alcançada pelas universidades. Assim, quanto mais unidades em funcionamento fora da cidade sede da universidade, menor a eficiência observada.

\section{Discussões}

Considerando o quantitativo de alunos de graduação e pós-graduação, bem como os indicadores de qualidade dos cursos oferecidos pelas universidades, pôde-se notar significativa ampliação da prestação de serviços à sociedade, principalmente no que tange à pós-graduação, que apresentou crescimento proporcionalmente superior. Importante destacar que, com a expansão do ensino superior, as universidades federais estão se tornando um pouco mais homogêneas em termos de número de matrículas. O programa possibilitou que as instituições menores expandissem a oferta de vagas, inclusive com a instalação de campi universitários em regiões antes não contempladas com instituições federais, tornando-as ligeiramente mais 
equiparadas, tendo em vista ainda existirem grandes diferenças de magnitudes entre elas, além do fato das grandes instituições também terem ampliado o número de matrículas.

Visando proporcionar a expansão do ensino superior, bem como o melhor aproveitamento da estrutura física e de recursos humanos existentes nas universidades federais, o Reuni teve como meta global a elevação da relação alunos em cursos presenciais por professor para dezoito. Observou-se, no entanto, que a média desse índice em 2008 foi de 12,3 alunos por professor, apresentando uma pequena redução durante os seis anos seguintes, atingindo um patamar ligeiramente superior $(12,5)$ apenas em 2015 , ou seja, em média, as universidades federais não foram capazes de atender a meta estabelecida.

Apesar da meta de dezoito alunos não ter sido alcançada, e da média de alunos por professor ter sofrido apenas um pequeno acréscimo, notou-se que a homogeneidade entre as universidades cresceu também em termos da composição desse indicador, cujos valores máximos e mínimos sugerem que aquelas instituições com menor relação aluno por professor buscaram ampliar o número de matrículas, otimizando a estrutura de recursos humanos. Por outro lado, as universidades com indicadores mais altos apresentaram redução na média de alunos por professor. Possivelmente, esse resultado pode ser explicado pelo fato de as universidades terem contratado docentes para lecionarem em cursos novos cujas vagas ainda não tenham sido totalmente preenchidas.

Não obstante o período estabelecido como meta do Reuni ter sido de cinco anos, a contar do início de cada plano, muitos cursos foram criados e novas vagas foram ofertadas nos anos subsequentes à implantação do programa, estendendo, portanto, o prazo necessário para o preenchimento dessas vagas, tendo em vista que os cursos criados recebem novas matrículas durante um período de dois a cinco anos, dependendo da sua duração, até que as primeiras turmas comecem a titular. Dessa forma, embora os cronogramas de contratações e investimentos na ampliação das universidades possam seguir dinâmicas distintas, é esperado que tais indicadores apresentem certa instabilidade até que as estruturas criadas alcancem a plena capacidade no atendimento de alunos.

Analisando o escore de eficiência gerado, notou-se que, em média, as universidades federais operam abaixo de suas capacidades, tendo em vista que, considerando o volume de recursos disponíveis para financiar suas atividades, elas poderiam gerar resultados significativamente maiores que os observados. Durante o período analisado, apenas no ano de 2009 observou-se uma queda no desempenho médio, com recuperação gradativa nos anos seguintes. Logo, não se puderam constatar variações representativas na média da eficiência observada, 
sugerindo que as modificações ocorridas em termos de investimentos públicos não geraram impactos diretos na eficiência. Possivelmente, os acréscimos no aporte de recursos do Governo Federal acompanharam o crescimento do quantitativo de alunos, ou a evolução dos indicadores de qualidade dos cursos de graduação e pósgraduação, ou ambos.

No ensejo de identificar os aspectos associados à eficiência das universidades federais brasileiras, buscou-se relacionar alguns indicadores apontados na literatura como condicionantes do desempenho de instituições de ensino superior com o escore de eficiência gerado, considerando o período de 2008 a 2015.

Assim, analisando a relação entre o indicador aluno por professor e o desempenho das universidades, verificou-se existir uma associação positiva entre eles. Quanto maior o número de alunos matriculados em relação ao quantitativo de professores, maior será a eficiência da universidade. Essa constatação pode ser explicada pela própria metodologia empregada para mensurar a eficiência, tendo em vista que o número de alunos matriculados é tratado como parte dos produtos gerados pelas universidades, e dessa forma, quanto maior a relação de alunos, maior será a sua eficiência. Apesar disso, ressalta-se que o acréscimo indiscriminado desse componente, sem os incrementos necessários para a manutenção das atividades da universidade, pode comprometer a qualidade dos serviços prestados, impactando negativamente os indicadores de qualidade dos cursos ofertados e, consequentemente, reduzindo o nível de eficiência. Portanto, o aumento no número de matrículas deve ser avaliado frente aos possíveis impactos nas condições de oferta dos cursos.

O elevado número de alunos por professor foi apresentado como uma das causas da baixa eficiência de universidades brasileiras no período de 2004 a 2008, independentemente do nível de dedicação às atividades de pós-graduação e pesquisa, conforme estudo realizado por Costa et al. (2012), em contradição aos achados da presente pesquisa. A explicação para a incongruência de resultados pode estar nos diferentes aspectos considerados para a mensuração da eficiência no referido estudo, sendo a relação de alunos formados por ingressantes (taxa de sucesso na graduação) e o conceito Capes utilizados como produtos das universidades, além de diferentes variáveis tratadas como insumos. Portanto, a adoção de procedimentos metodológicos diferentes não permite concluir que tais resultados representam, de fato, constatações contraditórias.

Oindicador alunos porfuncionáriotambém apresentou relação significativamente positiva com o escore de eficiência das universidades federais brasileiras, fato também justificado pelo aumento do número de matrículas representar maior produção das universidades; assim, cabem também as mesmas ressalvas feitas 
na análise da relação de alunos por professor. No mesmo estudo de Costa et al. (2012), a relação alunos por funcionário mostrou-se como um dos fatores que mais comprometeram a eficiência das universidades com menor atuação em pós-graduação e pesquisa, novamente contrapondo os resultados apresentados. No entanto, como já relatado, a adoção de critérios diferentes para mensurar a eficiência dificulta a comparação dos resultados.

O modelo de análise utilizado ainda apontou não existir relação significativa entre o escore de eficiência observado e o número de funcionários por professor da universidade, ou seja, com base nos dados utilizados, no período de 2008 a 2015, a proporção entre servidores técnicos e docentes não apresentou associação com o desempenho.

Na literatura, a qualificação do corpo docente foi apontada como determinante da eficiência em universidades da África do Sul, apresentando impacto positivo no desempenho das universidades (TAYLOR; HARRYS, 2004). No Brasil, Costa et al. (2012) relataram que o índice de qualificação do corpo docente foi um dos fatores que comprometeram a eficiência das instituições com pouca ou nenhuma atuação em pós-graduação, apresentando como possível justificativa a existência de professores qualificados que não estivessem sendo utilizados plenamente no desempenho de suas atividades acadêmicas.

Percebe-se, portanto, que a qualificação do corpo docente apresenta expectativas ambíguas em termos de sua relação com a eficiência das instituições de ensino superior, ao passo que, se por um lado os professores mais qualificados podem contribuir com a qualidade dos serviços prestados, por outro, a remuneração desses docentes geralmente é maior, podendo também onerar os gastos das universidades. No presente estudo, com base no recorte metodológico adotado, essa variável não se mostrou significativa, não sendo possível determinar a sua relação com a performance das universidades federais brasileiras no período analisado.

Alguns estudos buscaram analisar a eficiência considerando, separadamente, atividades de ensino e pesquisa, ou universidades com maior ou menor atuação em pós-graduação e pesquisa. Glass et al. (2006) constataram que as instituições do Reino Unido mais focadas em uma só atividade (ensino ou pesquisa) mostraramse mais eficientes. Sellers-Rubio, Mas-Ruiz e Casado-Díaz (2010) analisaram departamentos acadêmicos de uma universidade da Espanha e perceberam que, apesar da dedicação à pesquisa ter mostrado relação negativa com a eficiência no ensino, a eficiência no ensino mostrou-se complementar à eficiência na pesquisa. No Brasil, Costa et al. (2012) analisaram separadamente instituições com maior e menor atuação em pós-graduação e pesquisa, identificando diferentes fatores relacionados à baixa eficiência de ambas. 
Nesse sentido, no presente estudo, o grau de envolvimento da universidade com atividades de pós-graduação também foi inserido no modelo, no intuito de verificar se a maior dedicação das universidades às atividades de pós-graduação e, por consequência, de pesquisa, apresenta relação com o desempenho. No entanto, essa variável não se mostrou significativa na determinação da eficiência, impossibilitando conclusões acerca do impacto desse fator.

Analisando o tempo de existência das universidades e sua relação com a eficiência, o modelo proposto apontou não ser esse fator um condicionante do desempenho das universidades federais brasileiras, embora a literatura tenha registrado que, entre diversas instituições públicas de ensino superior de diferentes países europeus, as mais tradicionais, ou seja, aquelas em funcionamento há mais tempo, mostram-se mais eficientes (WolszCZAK-DERLACZ; PARTEKA, 2011).

Para avaliar a relação entre a dimensão da universidade e o nível de eficiência alcançado, utilizou-se o total de servidores como proxy do tamanho. Os resultados apontaram que as maiores universidades estão associadas aos maiores escores de eficiência, corroborando os achados de Glass et al. (2006) e Wolszczak-Derlacz e Parteka (2011), sugerindo a existência de economias de escala.

Por fim, o último fator considerado na análise refere-se a uma característica bastante relevante no atual cenário universitário brasileiro, tendo em vista as recentes políticas de expansão do ensino superior público. Trata-se da existência de universidades configuradas como multicampi, ou seja, instituições que possuem campus em funcionamento em diferentes municípios. Buscou-se analisar se a quantidade de campi fora da sede influencia no desempenho das universidades, e constatou-se que, quanto maior o número de unidades instaladas em municípios diferentes do campus sede, menor o nível de eficiência observado.

Esse resultado remete a algumas considerações a respeito do histórico de criação dos campi universitários, bem como acerca de aspectos relacionados à gestão acadêmica e administrativa dessas unidades. Por serem resultantes de uma recente política de ampliação da oferta de vagas no ensino superior, esses campi são compostos, em sua maioria, por cursos novos, portanto, ainda estão em processo de consolidação. Possivelmente, foram efetuados os investimentos e realizadas as contratações de servidores para atender demandas futuras, de forma que essas unidades podem estar temporariamente operando aquém de sua capacidade. Deve ser considerada ainda a existência de fatores que possam estar dificultando o preenchimento das vagas ofertadas ou prejudicando a permanência dos alunos matriculados, como os critérios de seleção adotados, a qualidade dos alunos ingressantes, as condições de oferta dos cursos, ou até mesmo a efetiva demanda dos cursos instalados nessas novas unidades. 
Importante investigar também a ocorrência de problemas de ordem administrativa nesses campi, tendo em vista as dificuldades que podem ser geradas em função das distâncias geográficas entre essas unidades e o campus sede. Nesse sentido, a estrutura administrativa das universidades multicampi deve ser bem definida e regulamentada, visando garantir que os processos de tomada de decisão ocorram de forma tempestiva e sem onerar os custos da instituição.

\section{Considerações finais}

Buscou-se desenvolver uma análise da eficiência relativa das universidades federais brasileiras que aderiram ao Reuni, bem como propor uma discussão sobre seus fatores condicionantes. A análise da eficiência permitiu verificar que, em média, as universidades federais brasileiras atuaram abaixo de seu potencial entre os anos de 2008 e 2015, sendo que, nesse período, apesar da pequena queda no desempenho observado, não se pôde identificar variações expressivas na eficiência. Apesar disso, é notável que, sob diversos aspectos, como a relação alunos por professor e a proporção do atendimento a alunos de graduação e pós-graduação, as universidades tornaram-se um pouco mais homogêneas.

Como limitações da pesquisa pode-se apontar que, em função da não disponibilidade de dados, o modelo empregado para mensurar a eficiência não contemplou variáveis que expressassem as atividades de pesquisa e extensão das universidades, embora se possa presumir que, ao serem incluídos os indicadores de qualidade, como o conceito médio da graduação e o conceito Capes para a pósgraduação, esteja-se ponderando, indiretamente, sobre as diversas atuações das instituições federais de ensino superior.

Além disso, verifica-se que a literatura ainda conta com poucos estudos sistemáticos sobre avaliação de políticas e programas universitários no Brasil, bem como sobre a análise de fatores determinantes do desempenho dessas instituições, o que faz com que o presente estudo tenha um caráter mais exploratório que explicativo. Nesse sentido, os resultados aqui apesentados têm como principal contribuição a discussão sobre alguns fatores condicionantes da eficiência, sem a pretensão de serem conclusivos.

Existem limitações também relacionadas ao período de análise. Ressalta-se que o estudo contemplou momentos em que ocorreram greves de servidores das universidades, nos anos de 2012 e 2015, implicando em descompassos nos calendários acadêmicos dessas instituições, tendo em vista que nem todas as universidades aderiram às paralisações. Igualmente, embora o período estabelecido como meta do Reuni tenha sido de cinco anos, as instituições ampliaram a oferta 
de cursos também nos anos posteriores à implantação do programa, estendendo, portanto, o prazo necessário para o preenchimento dessas vagas e a esperada otimização das estruturas criadas. Por isso, tendo em vista a contemporaneidade da expansão do ensino superior, sugere-se que sejam realizadas novas pesquisas contemplando períodos maiores, de forma a evidenciar os efeitos a longo prazo da ampliação desse nível de ensino.

\section{Referências bibliográficas}

AGASISTI, T. Performances and spending efficiency in higher education: a European comparison through non-parametric approaches. Education Economics, v. 19, n. 2, p. 199-224, 2011.

Ahn, T.; Charnes, A.; COOPER, W. Some statistical and DEA evaluations of relative efficiencies of public and private institutions of higher learning. Socio-Economic Planning Sciences, v. 22, n. 6, p. 259-269, 1988.

BARRA, C.; ZOTTI, R. A directional distance approach applied to higher education: an analysis of teaching-related output efficiency. Annals of Public and Cooperative Economics, v. 87, n. 2, p. 145-173, jun. 2016a.

. Measuring Efficiency in Higher Education: an empirical study using a bootstrapped data envelopment analysis. International Advances in Economic Research, v. 22, n.1, p. 11-33, feb. 2016b.

BELLONI, J. A. Uma metodologia de avaliação da eficiência produtiva de universidades federais brasileiras. 245 p. 2000. Tese (Doutorado em Engenharia de Produção) - Universidade Federal de Santa Catarina, Florianópolis, 2000. Disponível em: $\quad<$ https://repositorio.ufsc.br/bitstream/handle/123456789/78457/153160. pdf?sequence=1>. Acesso em: 22 jun. 2016.

BELLONI, I; MAGALHÃES, H. DE; SOUSA, L. C. DE. Metodologia de avaliação em políticas públicas. 3. ed. São Paulo: Cortez, 96 p., 2003.

BRASIL. Lei no 9.394/1996. Estabelece as diretrizes e bases da educação nacional. Disponível em: <http://www.planalto.gov.br/ccivil_03/leis/L9394.htm>. Acesso em: 22 jul. 2017.

. Decreto no 6.096/2007. Institui o Programa de Apoio a Planos de Reestruturação e Expansão das Universidades Federais - Reuni. Disponível em: <http://www.planalto.gov.br/ccivil_03/_Ato2007-2010/2007/Decreto/D6096. htm>. Acesso em: 29 jul. 2016.

BRASIL. Tribunal de Contas da União. Orientações para o cálculo dos indicadores de gestão: decisão plenária no 408/2002. Disponível em: <http://portal.mec.gov.br/ setec/arquivos/pdf/indicadores.pdf>. Acesso em: 05 jul. 2016.

BRASIL. Ministério da Educação/SESu. Programa de Apoio a Planos de Reestruturação e Expansão das Universidades Federais. Reuni 2008 - Relatório de primeiro ano, 30 de outubro de 2009. Disponível em: <http://portal.mec.gov.br/ 
index.php?option=com_docman\&view=download\&alias=2069-reuni-relatoriopdf\&category_slug=dezembro-2009-pdf\&Itemid=30192>. Acesso em 25 maio 2017.

BREU, T. M.; RAAB, L. R. Efficiency and perceived quality of the nation's "Top 25" national universities and national Liberal Arts colleges: an application of data envelopment analysis to higher education. Socio-Econ. Plann. Sci., v. 28, n. 1, p. 3345, 1994.

Charnes, A. COOPER, W. W., RHOdes, E. Measuring the efficiency of decision making units. European Journal of Operational Research, v. 2, p. 429-444, 1978.

CostA, E. M. et al. Eficiência e desempenho no ensino superior: uma análise da fronteira de produção educacional das IFES brasileiras. Rev. Econ. Contemp., v. 16, n. 3, p. 415-440, set./dez. 2012.

ECKLES, J. E. Evaluating the efficiency of top liberal arts colleges. Research in Higher Education, v. 51, p. 266-293, 2010.

FARRELL, M. J. The measurement of productive efficiency. Journal of the Royal Statistical Society, Series A, v.120, n. 3, p. 253-290, 1957.

FELDMANN, T., SOUZA, O. DE. A governamentalidade e o Exame Nacional de Desempenho De Estudantes - Enade. Avaliação, Campinas; Sorocaba, SP, v. 21, n. 3, p. 1.017-1.032, ago./nov. 2016.

FrançA, J. M. F. DE; Figueiredo, J. N. DE; LAPA, J. DOS S. A DEA methodology to evaluate the impact of information asymmetry on the efficiency of not-for-profit organizations with an application to higher education in Brazil. Ann Oper Res, v. 173, p. 39-56, 2010.

GLASS, J. C. et al. Implications of variant efficiency measures for policy evaluations in UK higher education. Socio-Economic Planning Sciences, v. 40, p. 119-142, 2006.

GoMES, A. P.; BAPTISTA, A. J. M. Dos S. Análise Envoltória de Dados: conceitos e modelos básicos. In: SANTOS, M. L. DOS; VIEIRA, W. DA C. (ed). Métodos Quantitativos em Economia. Viçosa MG: Editora UFV, 2004.

Greene, W. H. Econometric analysis. 3rd ed. New Jersey: Prentice-Hall, 1997.

Gujarati, D. N. Econometria básica. 3. ed. Tradução Ernesto Yoshida. São Paulo: Makron Books, 2000.

KIRJAVAINEN, T.; LOIKKANENTNA, H. A. Efficiency differences of finnish senior secondary schools: an application of DEA and Tobit analysis. Economics of Education Review, v. 17, n. 4, p 377-394, out. 1998.

MoreirA, N. P. et al. Fatores determinantes da eficiência dos programas de pósgraduação acadêmicos em administração, contabilidade e turismo. Avaliação, Campinas; Sorocaba, SP, v. 16, n. 1, p. 201-230, mar. 2011.

OBADIC, A.; ARISTOVNIK, A. Relative efficiency of higher education in Croatia and Slovenia: an international comparison. Amfiteatru Economic, v. 13, n. 30, jun. 2011. PORTO, C.; RÉGNIER, K. O ensino superior no mundo e no Brasil - Condicionantes, tendências e cenários para o horizonte 2003-2025: uma abordagem exploratória. 2003. Disponível em <http://portal.mec.gov.br/sesu/arquivos/pdf/ 
ensinosuperiormundobrasiltendenciascenarios2003-2025.pdf>. Acesso em: 08 de dez. 2014.

Sellers-Rubio, R., MAS-Ruiz, F. J.; CASAdo-DíaZ, A. B. University efficiency: complementariness versus trade-off between teaching, research and administrative activities. Higher Education Quarterly, v. 64, n. 4, p. 373-391, oct. 2010.

SoARES De Mello, J. C. C. B. et al. Avaliação qualitativa e quantitativa: uma metodologia de integração. Ensaio: avaliação e políticas públicas em educação. Rio de Janeiro, v. 9, n. 31, p. 237-251, 2001.

. Curso de análise de envoltória de dados. XXXVII SBPO - Simpósio Brasileiro de Pesquisa Operacional, 2005, Gramado. Anais do XXXVII SBPO, 2005.

TAYLOR, B.; HARRIS, G. Relative efficiency among South African universities: a data envelopment analysis. Higher Education, v. 47, p. 73-89, 2004.

Wolszczak-Derlacz, J.; PARTEKA, A. Efficiency of European public higher education institutions: a two-stage multicountry approach. Scientometrics, v. 89, p. 887-917, aug. 2011.

WoolDRIDGE, J. M. Introdução à econometria: uma abordagem moderna. São Paulo: Cengage Learning, 2013.

\section{Ney Paulo Moreira}

Universidade Federal de Viçosa - Campus de Rio Paranaíba-MG.Doutor em Administração pela Universidade Federal de Lavras.Professor Adjunto da Universidade Federal de Viçosa.

\section{Gideon Carvalho de Benedicto}

Instituição a que está vinculado: Universidade Federal de Lavras. Doutor em Controladoria e Contabilidade pela Universidade de São Paulo. Orientador de Mestrado e de Doutorado. Professor Associado da Universidade Federal de Lavras.

Francisval de Melo Carvalho

Universidade Federal de Lavras. Doutor em Administração de Empresas pela Universidade Presbiteriana Mackenzie. Orientador de Mestrado e de Doutorado. Professor Associado da Universidade Federal de Lavras. 